\title{
Large duct type invasive adenocarcinoma of the pancreas with microcystic and papillary patterns: a potential microscopic mimic of non-invasive ductal neoplasia
}

\author{
Pelin Bagci $^{1}$, Aleodor A Andea ${ }^{2}$, Olca Basturk ${ }^{3}$, Kee-Taek Jang ${ }^{4}$, Ipek Erbarut ${ }^{5}$ and Volkan Adsay \\ ${ }^{1}$ Department of Pathology, Rize University, School of Medicine, Rize, Turkey; ${ }^{2}$ Department of Pathology, University \\ of Alabama at Birmingham, Birmingham, AL, USA; ${ }^{3}$ Department of Pathology, Memorial Sloan-Kettering \\ Cancer Center, New York City, NY, USA; ${ }^{4}$ Department of Pathology, Samsung Medical Center, Sungkyunkwan \\ University School of Medicine, Seoul, Korea and ${ }^{5}$ Department of Pathology, Emory University, Atlanta, GA, USA
}

\begin{abstract}
A morphological variant of pancreatic ductal adenocarcinoma forming large ductal elements, large duct type ductal adenocarcinoma, is documented and its clinicopathological features are studied. These tumors may have microcystic and papillary growth patterns that closely mimic the non-invasive cystic and papillary pancreatic tumors such as: intraductal papillary-mucinous neoplasia, including the oncocytic variant, mucinous cystic neoplasms, and ducts involved by pancreatic intraepithelial neoplasia. In a review of 230 pancreatectomy specimens with ductal adenocarcinoma, 28 (8\%) cases of large duct type ductal adenocarcinomas were identified according to following criteria: more than $50 \%$ of the tumor sections available for examination contained infiltrative ducts with a diameter larger than $\mathbf{0 . 5} \mathbf{m m}$ or had a macroscopically identifiable microcystic pattern. Overall characteristics of large duct type ductal adenocarcinomas were not too different than those of conventional ductal adenocarcinomas, except that there was a slight female predominance in the former $(\mathrm{F} / \mathrm{M}=2.3)$. The mean age was 67 (vs 63 in conventional ductal adenocarcinomas; $P=0.015)$, and occurrence in the tail was slightly more common ( $40 \%$ vs $18 \%$ in conventional ductal adenocarcinomas; $P=0.006)$. Grossly, cysts measuring up to $1 \mathrm{~cm}$ was noted in 10 cases. Microscopically, large duct type adenocarcinomas were characterized by irregularly distributed large ducts with jagged edges, lined by columnar mucinous cells often having deceptively bland cytological features and variable degrees of papillomatosis. Stromal desmoplasia had a hypercellular quality (morphologically distinct from ovarian-like stroma) in four cases, and had a myxoid quality in others. KRAS oncogene mutation was identified in 9 out of 11 cases. Median, 1-year and 2-year survival rates were 16 months, $77 \%$ and $30 \%$, respectively, as opposed to 12 months, $52 \%$ and $30 \%$, respectively, in conventional ductal adenocarcinoma. In conclusion, it should be recognized that, some (8\%) pancreatic ductal adenocarcinomas exhibit a large duct pattern that may microscopically mimic non-invasive pancreatic tumors characterized by cystic and papillary patterns. They may be distinguished by the relatively smaller size of the cysts, irregularity of the duct contours, clustering of the ducts, presence of intraluminal neutrophils and granular debris, degree of cytological pleomorphism, and myxoid quality of the stroma. Clinical behavior appears to be slightly better than that of conventional ductal adenocarcinoma, which may be accounted by the well-differentiated nature of these tumors.

Modern Pathology (2012) 25, 439-448; doi:10.1038/modpathol.2011.181; published online 4 November 2011
\end{abstract}

Keywords: adenocarcinoma; large duct; microcystic; pancreas; papillary

Correspondence: Professor V Adsay, MD, Department of Pathology and Laboratory Medicine, Emory University Hospital, 1364 Clifton Road NE, Room H-180B, Atlanta, GA 30322, USA.

E-mail: volkan.adsay@emory.edu

This study was presented in part at the annual meeting of the United States and Canadian Academy of Pathology in, Washington DC in 2003.

Received 19 July 2011; revised 26 September 2011; accepted 28 September 2011; published online 4 November 2011
The vast majority of pancreatic malignancies are ordinary ductal adenocarcinomas, characterized by small tubular units. $^{1-3}$ However, a morphological variant of pancreatic ductal adenocarcinoma forming large ductal elements also do occur. ${ }^{4,5}$ This variant, which we refer to as large duct type ductal 
adenocarcinoma $^{6-9}$ is characterized by larger infiltrative ducts than the ordinary ductal adenocarcinomas, some with cystically dilated lumen and attenuated lining (Figure 1), and some others exhibiting papillary growth patterns. The remarkable ability of this variant to form cystic appearance was demonstrated by Kosmahl et $a l^{5}$ in their study of ductal adenocarcinomas with cystic features. In the past decade, with the better recognition of preinvasive ductal neoplasia including intraductal papillary-mucinous neoplasm, ${ }^{4,10,11}$ mucinous cystic neoplasm, ${ }^{12-15}$ and pancreatic intraepithelial neoplasia, ${ }^{16,17}$ which are also characterized microscopically by cystic ductal elements and papillary configuration, their challenging differential diagnosis from invasive large duct type ductal adenocarcinoma has also become more important.

In this study, the clinical and pathological characteristics of this morphological variant of ductal adenocarcinomas are investigated and compared with those of ordinary ductal adenocarcinomas. Further on, we also analyzed the pathological findings that may help in the challenging differential diagnosis of large duct type ductal adenocarcinomas from the non-invasive cystic and papillary tumors.

\section{Materials and methods}

\section{Selection of Cases}

All the pathology material available on 230 consecutive pancreatectomy specimens with invasive ductal adenocarcinoma identified in the institutional surgical pathology files were retrieved and reviewed to identify the cases exhibiting a large duct type pattern. All cases composed predominantly of large infiltrative duct units were included in large

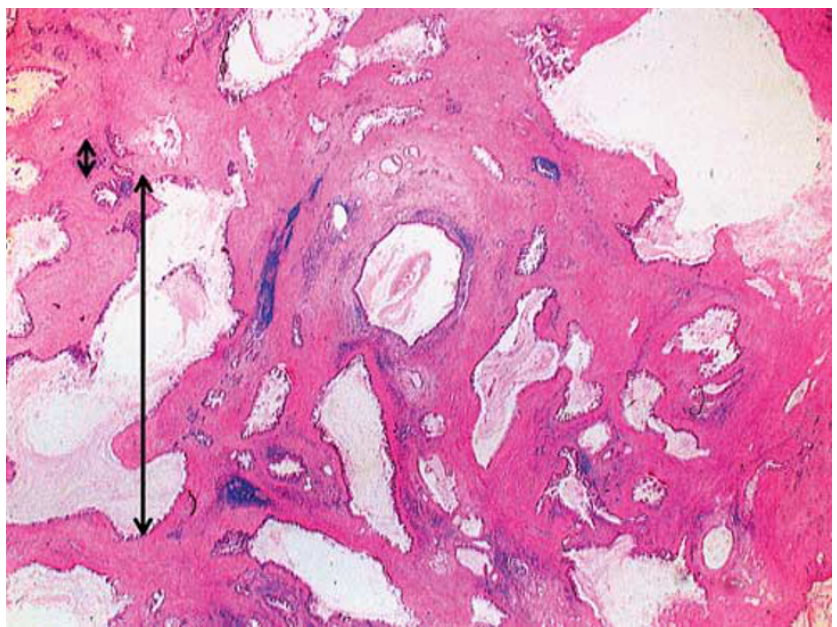

Figure 1 Atypical glands with cystic lumen and attenuated lining, typical for large duct type ductal adenocarcinoma (pancreatic islets are marked by arrow heads for comparison of the size). duct type ductal adenocarcinoma category. To provide consistency and reproducibility in identifying this pattern, we categorized a case as large duct type, if more than $50 \%$ of the tumor sections available for examination contained infiltrative duct units with a diameter larger than $0.5 \mathrm{~mm}$ or had a macroscopically identifiable microcystic pattern. Ductal adenocarcinoma often forms larger stretched units when infiltrates into the duodenal musculature. This was not accounted towards the $50 \%$ requirement to include a case in this study. We identified 28 cases that fulfilled these criteria. The patients' charts were reviewed to obtain demographic and clinical data (distant metastases, survival). Median follow-up time was 16 months (range 3-54 months). A total of 25 out of 28 patients died of disease. The size of the tumor, presence of positive margins, presence of lymph node metastases, vascular and perineural invasion, and local extension were assessed by consulting the pathology reports and also by reviewing the original tissue sections. The degree of stromal desmoplasia and cellularity were also recorded. All tumors were staged according to the AJCC recommendations. ${ }^{18}$

\section{Immunohistochemistry}

Immunoperoxidase stains were performed using the avidin-biotin complex technique. We investigated immunohistochemical expression of p53 (Vector, Burlingame, CA, USA; Clone DO-7, Dilution-1:80) and her2-neu (Zymed San Francisco, CA, USA; Clone $c$-erb-B2, Dilution-1:10) in 11 cases. In the four cases that demonstrated a hypercellular peritumoral stromal response, we performed immunohistochemical stains for desmin (Dako, Carpinteria, CA, USA; Clone D33, Dilution-1:800), actin (Dako; Clone HHF35, Dilution-1:100), estrogen (Dako; Clone 1D5, Dilution-1:50), and progesterone receptors (Dako; Clone PgR636, Dilution-1:400) to evaluate the stromal expression of these antigens.

Negative and positive controls were included with each batch of cases tested. We evaluated the percentage of cells staining with every antibody. For statistical analysis, all cases exhibiting staining in more than $20 \%$ of the cells were considered positive.

\section{KRAS Mutation Analysis}

In 12 cases where frozen tissue specimens were available, we investigated the presence of mutations in codon 12 of the KRAS gene. The method used was previously described elsewhere. ${ }^{19}$ Briefly, after DNA extraction, a PCR reaction was carried out in a $50 \mu \mathrm{l}$ of reaction mixture using specific primers. The primer set consisted of $5^{\prime}$-ATGACTGAATAT AAACTTGT-3' (forward) and 5'-CTATTGTTGGATC ATATT-3' (reverse). We used a DNA thermal cylinder (Cetus-Perkin Elmer, Norwalk, CT, USA) for 35 
cycles to complete the amplification. Each cycle of amplification consisted of 1 min of denaturation at $94^{\circ} \mathrm{C}, 1 \mathrm{~min}$ of annealing at $57^{\circ} \mathrm{C}$, and $2 \mathrm{~min}$ of polymerization at $72^{\circ} \mathrm{C}$. After the last cycle, polymerization was continued for an additional $7 \mathrm{~min}$ at $72^{\circ} \mathrm{C}$.

Slot-blot Southern analysis of PCR-amplified samples were carried out using ${ }^{32} \mathrm{P}$-labeled oligonucleotides probe panel (a wild-type probe and six specific probes for each base-pair mutation) following standard procedures. ${ }^{19}$

\section{Comparison with Ordinary Ductal Adenocarcinomas}

To compare the clinicopathological characteristics of large duct type ductal adenocarcinoma with ductal adenocarcinoma, we used a control group composed of 202 patients from the files of our institutions that underwent a pancreatectomy procedure, and where pathology revealed ordinary ductal adenocarcinoma. In this control series, the median follow-up time was 10 months (range 1-117 months). A total of 123 out of 202 patients died of disease. Mean age at presentation and tumor size were compared using independent samples $t$-tests. Differences in the distribution of gender, race, and TNM stage were assessed utilizing $\chi^{2}$-statistic tests. A $P$-value of $<0.05$ was regarded as statistically significant. Survival data was evaluated by constructing a Kaplan-Meier model, and the differences between large duct type ductal adenocarcinoma and ductal adenocarcinoma assessed by computing log-rank statistic test. Data on immunohistochemical expression of p53 and her2-neu were available in 52 and 49 cases, respectively. In addition, mutational analysis in codon 12 of KRAS was performed in 47 cases. The results of these molecular and immunohistochemical analysis were compared with those of large duct type ductal adenocarcinoma.

\section{Results}

\section{Clinical Features}

Clinical characteristics of the large duct type ductal adenocarcinoma cases compared with ordinary ductal adenocarcinomas are summarized in Table 1. The mean age of the patients at presentation was 67 years (range 51-87 years), slightly higher than the age of patients with ordinary ductal adenocarcinoma, 63 years (range 22-86 years), $P=0.01$. Whereas in the large duct type ductal adenocarcinoma series, the mean age at presentation for males was higher than for females $(M=70$, $\mathrm{F}=66$ years, $P=0.2$ ), in the control group of ordinary ductal adenocarcinoma cases, the opposite was true $(\mathrm{M}=61, \mathrm{~F}=64$ years, $P=0.1)$.

In contrast with ordinary ductal adenocarcinoma, which afflicted females and males equally $(\mathrm{F}=53 \%$,
Table 1 Clinicopathological features of large duct type adenocarcinoma compared with ordinary ductal adenocarcinoma cases

\begin{tabular}{|c|c|c|c|}
\hline & $\begin{array}{l}\text { Large duct type } \\
\text { adenocarcinoma, } \\
\text { (28 cases) }\end{array}$ & $\begin{array}{l}\text { Ordinary ductal } \\
\text { adenocarcinoma, } \\
\text { (202 cases) }\end{array}$ & $\begin{array}{c}\mathrm{P}- \\
\text { value }\end{array}$ \\
\hline Mean Age (years) & 67 & 63 & 0.01 \\
\hline \multicolumn{4}{|l|}{ Gender } \\
\hline Female & $19(70 \%)$ & $105(53 \%)$ & \multirow[t]{2}{*}{0.08} \\
\hline Male & $8(30 \%)$ & $93(47 \%)$ & \\
\hline \multicolumn{4}{|l|}{ Tumor location } \\
\hline Head & $16(60 \%)$ & $154(82 \%)$ & 0.006 \\
\hline Tail & $11(40 \%)$ & $33(18 \%)$ & - \\
\hline $\begin{array}{l}\text { Median survival } \\
\text { (months) }\end{array}$ & 16 & 12 & 0.6 \\
\hline 1-year survival & $77 \%$ & $52 \%$ & - \\
\hline 2-year survival & $30 \%$ & $30 \%$ & - \\
\hline Tumor size $(\mathrm{cm})$ & 4.1 & 3.4 & 0.1 \\
\hline \multicolumn{4}{|l|}{ Stage } \\
\hline I & $4 / 27(15 \%)$ & $25 / 168(15 \%)$ & 0.02 \\
\hline II & $6 / 27(22 \%)$ & 49/168 (29\%) & - \\
\hline III & $12 / 27(44 \%)$ & $88 / 168(52 \%)$ & - \\
\hline IV & $5 / 27(19 \%)$ & $6 / 168(4 \%)$ & - \\
\hline \multicolumn{4}{|l|}{ Extent of tumor ${ }^{6}$} \\
\hline $\mathrm{EP}$ & $21 / 25(84 \%)$ & 109/119 (92\%) & 0.2 \\
\hline Duodenum & $8 / 25(32 \%)$ & NA & - \\
\hline Stomach + Colon & $3 / 25(12 \%)$ & NA & - \\
\hline $\begin{array}{l}\text { Cases with LN } \\
\text { metastasis }\end{array}$ & $16 / 23(70 \%)$ & $87 / 140(62 \%)$ & 0.5 \\
\hline $\begin{array}{l}\text { Average number of } \\
\text { metastatic LN }\end{array}$ & 2 & 3 & 0.3 \\
\hline Perineural invasion & $21 / 24(88 \%)$ & NA & - \\
\hline Vascular invasion & $5 / 23(22 \%)$ & NA & - \\
\hline Positive margins & $6 / 25(24 \%)$ & NA & - \\
\hline
\end{tabular}

Abbreviations: EP, direct extrapancreatic extension including duodenum, bile duct, peripancreatic adipose tissue; LN, lymph node; NA, not available.

$\mathrm{M}=47 \%$ ), there was a slight female preponderance in large duct type ductal adenocarcinoma $(\mathrm{F}=70 \%$, $\mathrm{M}=30 \%), P=0.08$.

Large duct type ductal adenocarcinomas tended to involve the tail of the pancreas significantly more frequent than ordinary ductal adenocarcinomas (40\% vs $18 \%, P=0.006$ ).

A comparative analysis of survival in large duct type ductal adenocarcinoma and ductal adenocarcinoma cases is presented in Figure 2. Within the large duct type ductal adenocarcinoma series, median survival after diagnosis, and 1-year and 2-year survival rates were 16 months, 77 and $30 \%$, respectively, as opposed to 12 months, $52 \%$ and $30 \%$, respectively, for ordinary ductal adenocarcinoma. A log-rank statistical test showed no significant differences in survival $(P=0.6)$. Within the large duct type ductal adenocarcinoma group, patients with tumors located in the head of the pancreas demonstrated a significantly longer median survival (19 months) than those with tumors arising from the tail (13 months), $P=0.006$. 


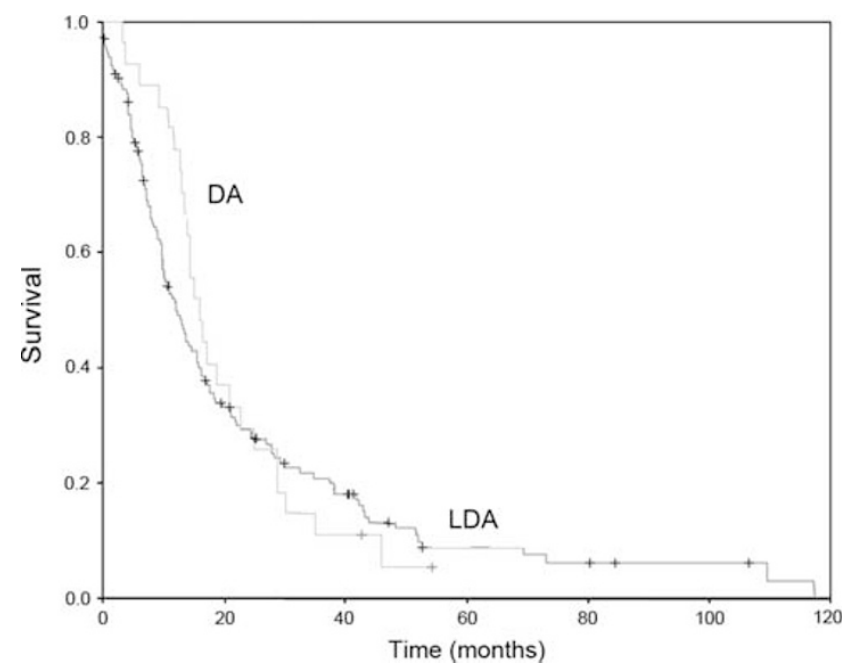

Figure 2 Comparative analysis of survival in large duct type ductal adenocarcinoma (LDA) and conventional ductal adenocarcinoma (DA).

\section{Macroscopic Features}

Macroscopically, the large duct type ductal adenocarcinomas had a firm consistency, infiltrative borders and a white color. In 10 cases, a microcystic pattern with cysts measuring from millimeters to up to $1 \mathrm{~cm}$ was noted (Figure 3). One case had cystic degeneration forming a 7-cm cavity. Gross papillary frond formation was not seen in any of the cases.

\section{Microscopic Features}

Microscopically, large duct type ductal adenocarcinomas were characterized by irregularly distributed cystic ducts, lined by columnar mucinous cells. The diameter of the majority of these ducts ranged from $0.5 \mathrm{~mm}$ to $1 \mathrm{~cm}$ (Figure 4). In most cases, areas typical of large duct type ductal adenocarcinoma ducts were associated with areas of usual ductal adenocarcinoma. According to our criteria in selecting the large duct type ductal adenocarcinoma cases, the usual ductal adenocarcinoma constituted less than $50 \%$ of the total tumor volume. In many areas, the cells were well-polarized, with focal pseudostratification and bland cytology. In general, the invasive glands were focally lined by foamy cells (characteristic of the 'foamy gland variant' of pancreatic adenocarcinoma), which had abundant, pale, foamy/microvesicular cytoplasm in which the vesicles were small and uniform, and basally located, irregular, and wrinkled nuclei. The cytoplasmic borders were often distinct and chromophilic apical condensation of the cytoplasm, forming a thin, distinct band lining the apical surface like a cuticle (brush border-like zone) was also present in these cells (Figure 4, inset). A papillary growth pattern, with focal cribriform areas, was present in variable degrees; however, it was not as exuberant as

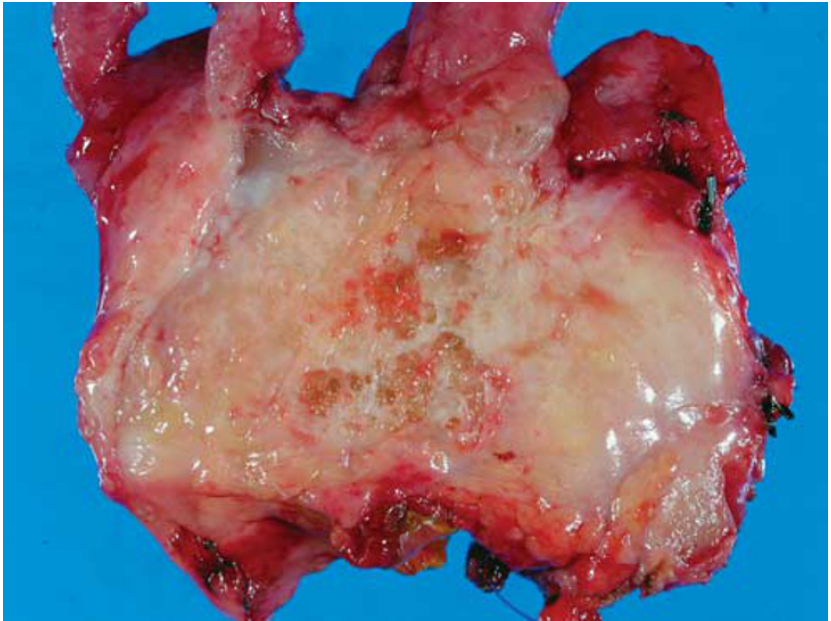

Figure 3 Gross appearance of large duct type ductal adenocarcinoma. A microcystic pattern with cysts measuring from millimeters up to $1 \mathrm{~cm}$.

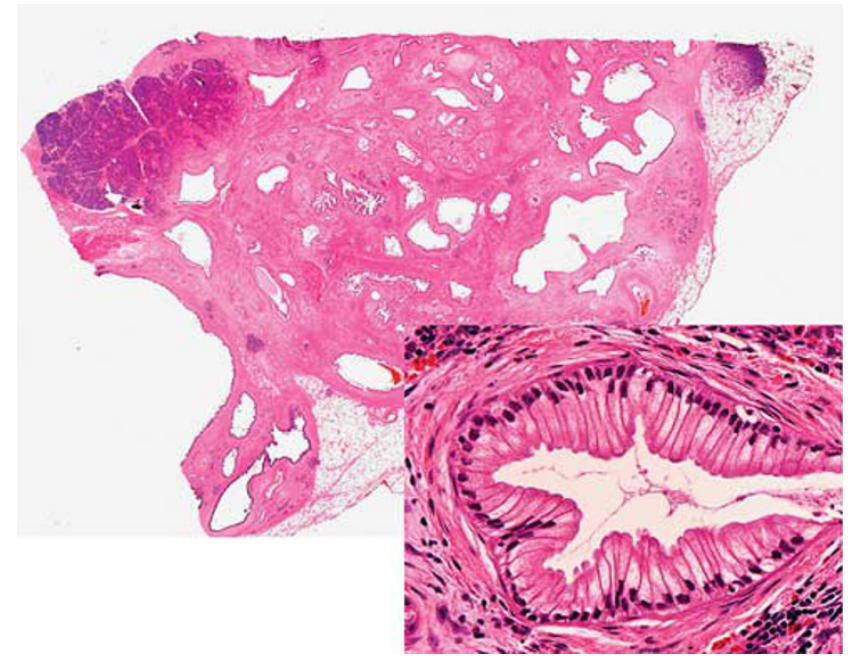

Figure 4 The diameter of the majority of these ducts ranged from $0.5 \mathrm{~mm}$ to $1 \mathrm{~cm}$. Invasive glands lined by foamy cells that are characteristic of the 'foamy gland variant' of pancreatic ductal adenocarcinoma (inset).

in the intraductal papillary mucinous neoplasms or intraductal oncocytic papillary neoplasms (Figure 5), and certainly not reaching to a level to form grossly detectable nodules. Hypercellular stroma was present in four cases, resembling the ovarian-like stroma of mucinous cystic neoplasms, but having a more desmoplastic appearance, rather than the wavy appearance of ovarian-like stroma (Figure 6a), showing strong expression of actin (Figure 6b). The presence of intraluminal collection of neutrophils and granular debris were also common occurrences in large duct type ductal adenocarcinoma. In addition, in some cases, especially those in the tail, there were granuloma-like collections of macrophages cuffing the invasive ducts, including focal microcalcifications, and focally obliterating the epithelium. 
We found that certain areas from sections of largeduct tumors containing cystically dilated invasive ducts, often resemble the histological pattern of noninvasive cystic or papillary tumors, such as intraductal papillary-mucinous neoplasm (Figure 7a), including the oncocytic variant and mucinous cystic neoplasm (Figure 7b). Other large duct type ductal adenocarcinomas that exhibited prominent papillary formation histologically mimicked ducts involved by pancreatic intraepithelial neoplasia (Figure 7c). Intraluminal mucin formation was prominent in many cases, and in some, it extravasated into the stroma, resembling invasive mucinous colloid carcinomas.

The pathological findings of large duct type ductal adenocarcinoma compared with ordinary ductal adenocarcinoma cases are presented in Table 1. No

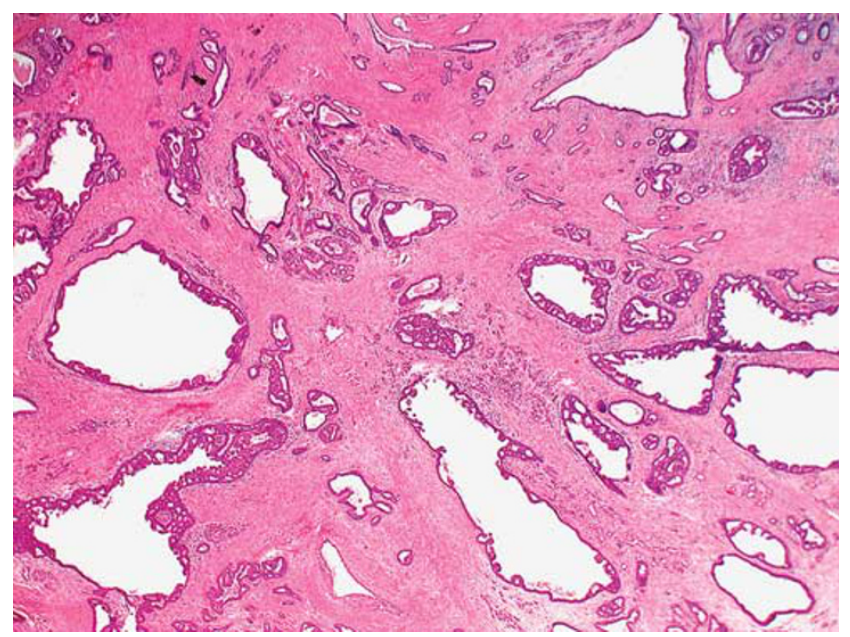

Figure 5 Papillary growth pattern with focal cribriform areas was present in variable degrees, but not as prominent as in intraductal papillary mucinous neoplasm or intraductal oncocytic papillary neoplasm. statistical significant differences between large duct type ductal adenocarcinoma and ordinary ductal adenocarcinoma were identified in mean tumor size ( $4 \mathrm{~cm}$ vs $3 \mathrm{~cm}$, respectively, $P=0.1$ ). The distribution of TNM stages was relatively similar in large duct type ductal adenocarcinoma as compared with the ordinary ductal adenocarcinoma cases. The only difference identified was for stage IV tumors that were more frequent in large duct type ductal adenocarcinoma versus ductal adenocarcinoma groups $(19 \%$ vs $4 \%, P=0.02)$. Perineural invasion was identified in 21 out of 24 cases $(88 \%)$ cases, often with the invading duct having a deceptively benign appearance. Large duct type ductal adenocarcinoma tumors demonstrated direct extrapancreatic extension in 21 out of 25 cases (84\%; Figure 8), not very different from the incidence of extrapancreatic extension observed in the control ductal adenocarcinoma group, 109 out of 119 cases $(92 \%), P=0.2$. Although the average number of metastatic lymph nodes was almost the same with ductal adenocarcinoma, metastatic carcinomas in lymph nodes from patients with large duct type ductal adenocarcinoma showed variable patterns. Although small tubular pattern often predominant, in some cases, large duct pattern closely mimicking non-invasive neoplasia was displayed even in the metastatic lymph node (Figure 9).

A recurring feature we observed during the review process of pancreatectomy specimens was that in some cases of ordinary ductal adenocarcinoma, the invasive units became significantly larger and elongated when they invaded the duodenal muscularis propria, only to revert to smaller sizes (similar to ductal adenocarcinoma) as they reach the duodenal submucosa. As the amount of tumor involving the duodenal muscularis propria represented only a small component $(<50 \%)$ of the total tumor volume, these cases were not included in the large duct type ductal adenocarcinoma group.
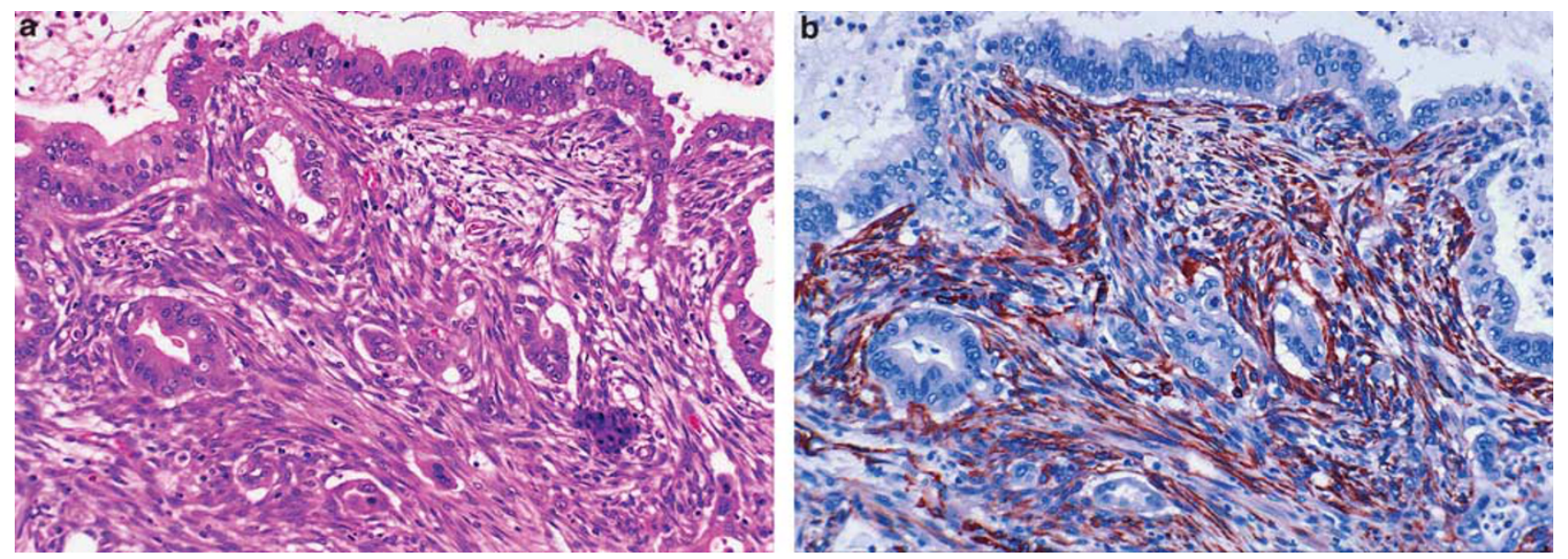

Figure 6 Hypercellular stroma was present in four cases, resembling the ovarian-like stroma of mucinous cystic neoplasms, but having a more desmoplastic appearance, rather than the wavy appearance of ovarian-like stroma (a), showing strong expression of actin (b). 

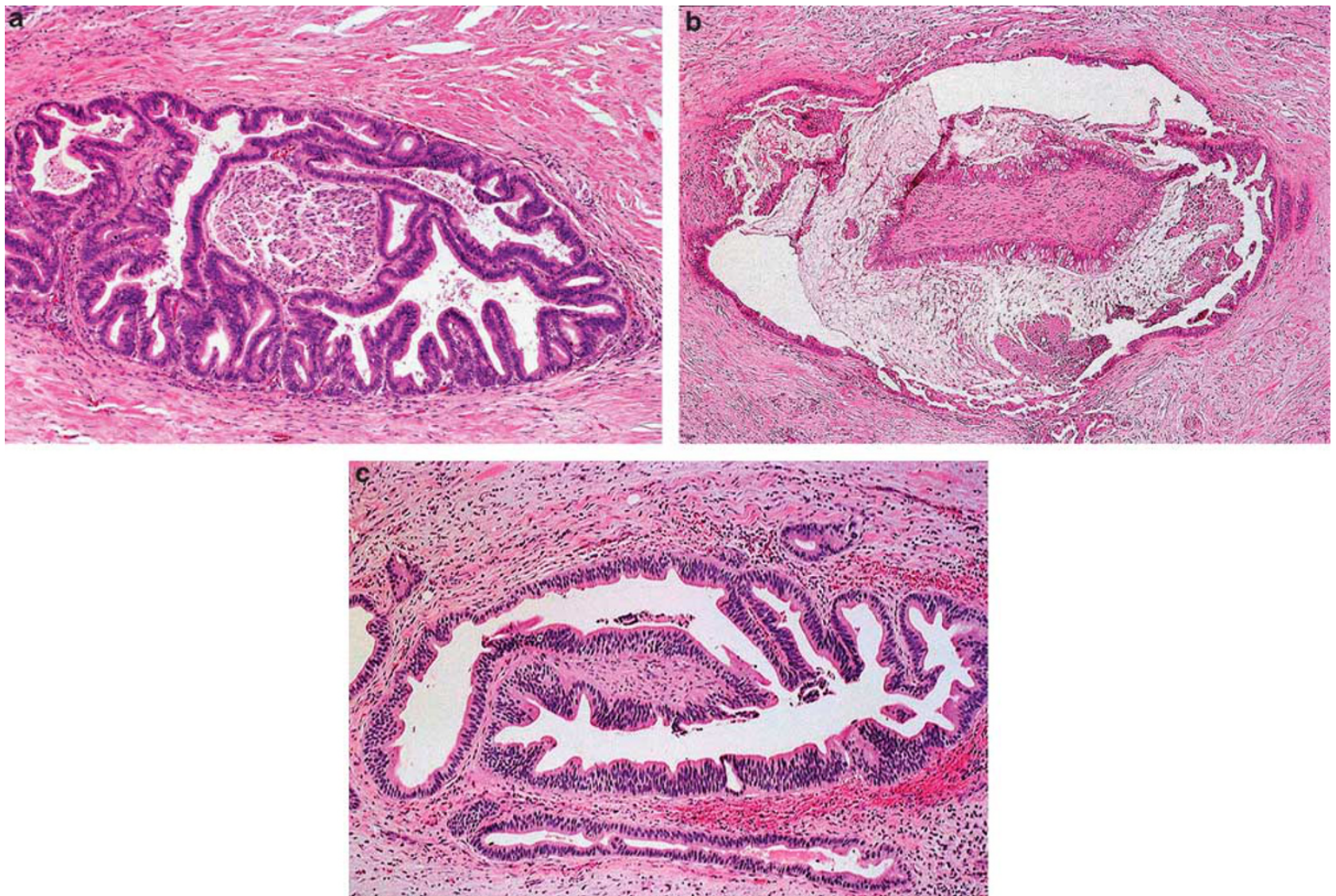

Figure 7 Large duct type ductal adenocarcinoma often resembles the histological pattern of non-invasive cystic or papillary tumors: intraductal papillary mucinous neoplasm (a), and mucinous-cystic neoplasm (b). Some large duct type ductal adenocarcinomas exhibited prominent papillary formation, histologically mimics ducts involved by pancreatic intraepithelial neoplasia (c).

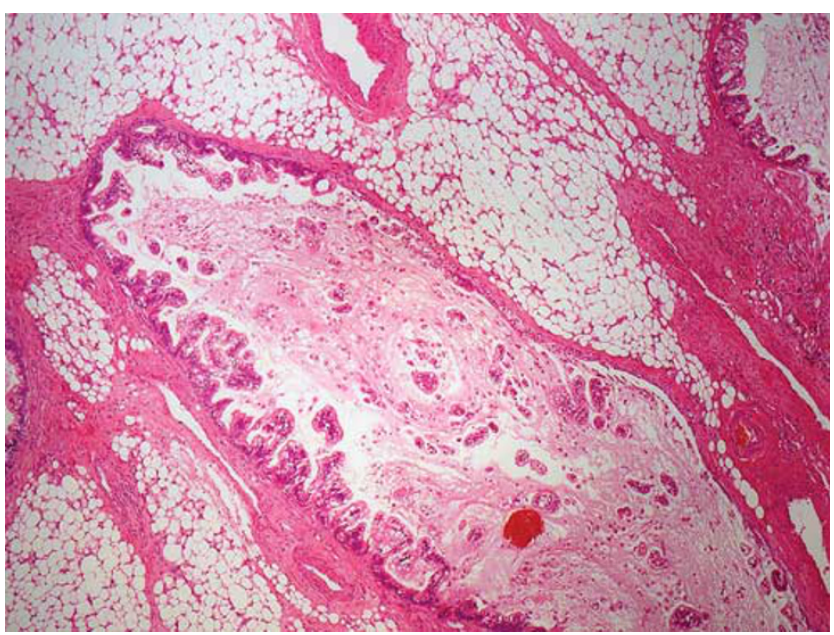

Figure 8 Direct extrapancreatic extension of large duct type ductal adenocarcinoma.

\section{Immunohistochemical and KRAS Mutation Analysis Results}

The results of the immunohistochemical studies are presented in Table 2. Overexpression of p53 and her2-neu at the immunohistochemical level was

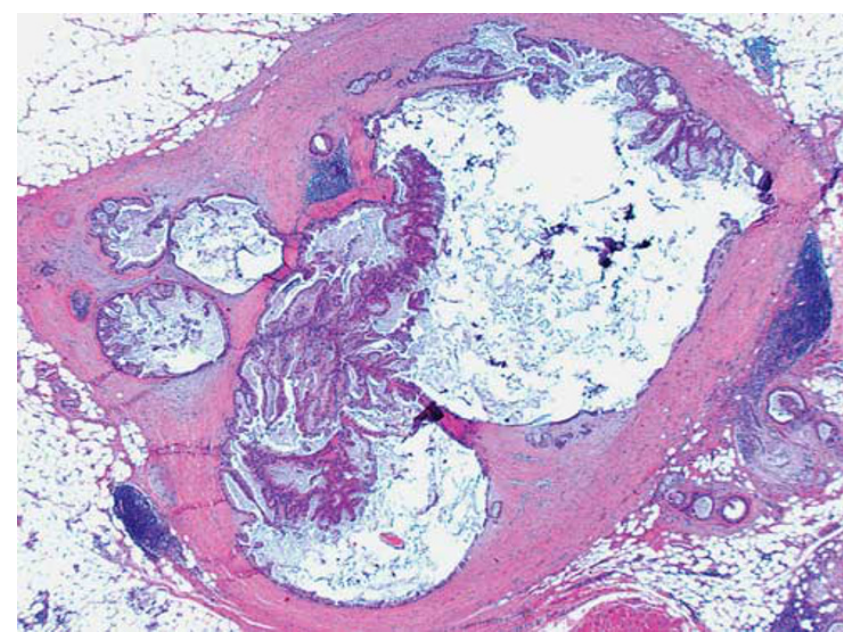

Figure 9 Metastatic carcinomas in lymph nodes from patients with large duct type ductal adenocarcinoma showed variable patterns. Although small tubular pattern was often predominant; in some cases, large-duct pattern closely mimicking non-invasive neoplasia was displayed even in the metastatic lymph node.

observed in $73 \%$ and $82 \%$, respectively. Analysis of the KRAS gene demonstrated mutation involving the codon 12 in 9 out of 11 cases (82\%). No statistical 
Table 2 Immunohistochemistry and mutational analysis of KRAS gene (codon 12) results

\begin{tabular}{|c|c|c|c|c|c|c|c|}
\hline & p53 (\%) & her2-neu (\%) & $\operatorname{Actin}^{\mathrm{a}}(\%)$ & $\operatorname{Desmin}^{\mathrm{a}}(\%)$ & $E R^{\mathrm{a}}(\%)$ & $P R^{\mathrm{a}}(\%)$ & KRAS (\%) \\
\hline Large duct type adenocarcinomas & $8 / 11(73)$ & 9/11 (82) & $4 / 4(100)$ & $0 / 4(0 \%)$ & $0 / 4(0)$ & $0 / 4(0)$ & $9 / 11(82)$ \\
\hline Ordinary ductal adenocarcinomas & $31 / 52(60)$ & $36 / 49(74)$ & Not done & Not done & Not done & Not done & $36 / 47(77)$ \\
\hline$P$-value & 0.5 & 0.7 & & & & & 0.5 \\
\hline
\end{tabular}

${ }^{\mathrm{a}}$ Staining was read in hypercellular stroma surrounding carcinomatous glands.

Table 3 Differential diagnosis criteria between invasive large duct type adenocarcinoma and non-invasive tumors

\begin{tabular}{|c|c|c|c|c|}
\hline & $\begin{array}{l}\text { Large duct type } \\
\text { adenocarcinoma }\end{array}$ & $\begin{array}{l}\text { Mucinous } \\
\text { cystic neoplasm }\end{array}$ & $\begin{array}{l}\text { Intraductal papillary } \\
\text { mucinous neoplasm }\end{array}$ & $\begin{array}{l}\text { Pancreatic intraepithelial; } \\
\text { neoplasm (PanIN) }\end{array}$ \\
\hline \multicolumn{5}{|l|}{ Ducts } \\
\hline Contours & Jagged, irregular & Smooth, round & Smooth, round & Smooth, undulating \\
\hline Distribution & $\begin{array}{l}\text { Irregular, focally clustered, } \\
\text { but separated by stroma }\end{array}$ & Regular, closely packed & $\begin{array}{l}\text { Regular, separated by } \\
\text { pancreatic tissue or fibrosis }\end{array}$ & Regular \\
\hline Tributary ducts & Absent & $\begin{array}{l}\text { May have attached } \\
\text { daughter microcysts }\end{array}$ & Present & Present \\
\hline Contents & $\begin{array}{l}\text { Intraluminal mucin, } \\
\text { neutrophils and } \\
\text { necrotic debris }\end{array}$ & & $\begin{array}{l}\text { Minimal mucin } \\
\text { precipitation }\end{array}$ & $\begin{array}{l}\text { Clear in low-grade PanIN } \\
\text { May be necrotic in } \\
\text { high-grade PanIN }\end{array}$ \\
\hline Cysts & May be microcystic & Megacystic & Microcystic or megacystic & Microcystic \\
\hline \multirow[t]{2}{*}{ Papillae } & $\begin{array}{l}\text { When present, they are } \\
\text { microscopic }\end{array}$ & $\begin{array}{l}\text { Grossly visible papillary } \\
\text { nodules in some cases }\end{array}$ & $\begin{array}{l}\text { Grossly visible } \\
\text { papillary nodules in } \\
\text { many cases }\end{array}$ & Mucosal folds \\
\hline & & & & $\begin{array}{l}\text { Rare abortive short } \\
\text { papillae }\end{array}$ \\
\hline \multicolumn{5}{|l|}{ Stroma } \\
\hline Cellular & $\begin{array}{l}\text { Occasional desmoplastic } \\
\text { type } \\
\text { Desmin (-) } \\
\text { Actin ++ } \\
\text { ER/PR (-) }\end{array}$ & $\begin{array}{l}\text { Ovarian-like } \\
\text { Desmin + } \\
\text { Actin ++ } \\
\text { ER/PR + }\end{array}$ & Absent & Absent \\
\hline Neural invasion & May be present & Absent & Absent & Absent \\
\hline
\end{tabular}

Abbreviations: ER, estrogen receptor; PR, progesteron receptor.

significant differences between large duct type ductal adenocarcinoma and ductal adenocarcinoma were identified in immunohistochemical expression of p53, her2-neu, and mutational analysis of codon 12 of KRAS. In the four cases that exhibited a peritumoral hyperplastic stroma, the stromal cells demonstrated strong expression of actin (Figure 6b), and negativity for desmin, and estrogen and progesterone receptors. This pattern of staining is different from the ovarian-like stroma surrounding the cystic spaces of mucinous cystic neoplasm, which are usually positive for desmin, as well as estrogen/ progesterone receptors.

\section{Discussion}

Majority of tumors involving the pancreas is of ductal origin. By far, the most common of these is ordinary (pancreatobiliary type) ductal adenocarci- noma, which is characterized by small, irregularly shaped ductal elements infiltrating in a desmoplastic stroma. ${ }^{1,20}$ As seen in this study, however, in $7 \%$ of the cases, pancreatic adenocarcinomas are composed predominantly of more dilated malignant ducts, forming a microcystic pattern, and that this cystic change can be very striking ${ }^{5}$ and even form a pattern that can be mistaken as a cystic preinvasive neoplasm both radiologically and pathologically. ${ }^{4-9}$ We refer to this morphological variant of invasive ductal adenocarcinoma as large duct type ductal adenocarcinomas.

In the cases that imaging findings were available for evaluation, it appeared that most cases of large duct type ductal adenocarcinoma were thought to be predominantly solid tumors, with the exception of those cases with true megacystic component. However, we have seen examples that were mistaken as IPMN by experienced radiologist with no IPMN component, despite careful and extensive sampling. 
Although distribution of the large ducts in ductal adenocarcinoma varied from case to case, in most cases, the large duct type ductal adenocarcinoma pattern was fairly uniform in a given area with only few interspersed smaller units. In this study, we selected and evaluated the cases of pancreatic adenocarcinomas in which the large invasive ductal units represented more than $50 \%$ of the total examined tumor. For the purpose of this investigation, we defined arbitrarily a duct as 'large' when its diameter exceeded $0.5 \mathrm{~mm}$. In some cases, the diameter of the ducts in the large duct type ductal adenocarcinoma component measured up to $1 \mathrm{~cm}$, creating a microcystic appearance that was occasionally detectable at macroscopic level. In contrast, majority of the invasive units in ordinary ductal adenocarcinoma have a diameter in the range of $0.05-0.2 \mathrm{~mm}$.

The morphological distinction between pancreatic adenocarcinoma and inflammatory reactive changes is a well-known challenge, due to the fact that ductal adenocarcinoma may exhibit welldifferentiated patterns with bland cytological features, and conversely, sections from glands injured by chronic pancreatitis may display significant epithelial atypia and fibrosis. ${ }^{21}$ In our series, the large ducts, although malignant, were in most cases lined by columnar or cuboidal mucinous cells with relatively bland cytological features and in some cases with prominent papillary architecture. Focally, the invasive glands were sometimes lined by foamy cells that are characteristic of the 'foamy gland variant' of pancreatic adenocarcinoma. ${ }^{4}$

Large duct type ductal adenocarcinoma closely resembles non-invasive pancreatic tumors that have a cystic and/or papillary pattern, namely intraductal papillary mucinous neoplasia (including the oncocytic ones), mucinous cystic neoplasms and pancreatic intraepithelial neoplasia. The invasive character of these malignant large ducts might be rather unapparent, especially when dealing with small biopsies. As there are no basal cells or a welldefined basement membrane around the native ducts of pancreas (unlike breast and prostate), we cannot rely on their demonstration by immunoperoxidase or histochemical stains to differentiate benign from malignant units. Therefore, the morphological distinction is often not obvious, and an integrated approach that takes into consideration the overall distribution of the ductal units, as well as the cytological features (especially the presence of foamy cells) ought to be utilized to arrive at the correct diagnosis. Table 3 presents the features that aid in differentiating invasive large ducts from their non-invasive mimics. Coexistence of a more ordinary small tubular component with otherwise similar morphology between large ducts is a good clue to the diagnosis. When found, the presence of perineural invasion makes a definite diagnosis of invasion. Invasive ductal elements lack identifiable tributary ducts, which are typically present in noninvasive cystic tumors. Large duct type ductal adenocarcinomas frequently have a desmoplastic appearance of the stroma with occasional myxoid quality. Another important feature of the malignant large ducts is their irregular contour, with jagged edges (in contrast with the smooth, round contour of non-invasive tumors) and tendency to be irregularly distributed. If the entirety of the tumor is available for examination, then the distinction of large duct type ductal adenocarcinoma from non-invasive processes such as IPMN is relatively straightforward, based on the sheer amount of the ducts, their jagged contours, and their association with foci of more conventional small tubular pattern of ductal adenocarcinoma. However, in biopsy specimens, frozen sections and the sections away from the main tumor, this distinction can become highly challenging (Figures 5 and $7 \mathrm{a}$ ). In that case, the contour irregularities, the presence of necrotic/neutrophilrich debris, and if present, the cytological findings of foamy gland variant would have to be relied upon for this distinction.

Periductal granulomas are also noted as relatively specific features. As distinct from the present knowledge, ${ }^{5}$ occasionally, the cellularity of the stroma resembles the ovarian-like stroma of mucinous cystic neoplasia; however, it lacks its wavy appearance and is negative for desmin and estrogen and progesterone receptors. This feature is important in terms of morphological distinction between large duct type ductal adenocarcinomas and mucinous cystic neoplasia.

Clinical behavior of large duct type ductal adenocarcinoma appears to be slightly better than it is for ordinary ductal adenocarcinomas. The difference in prognosis may be explained by the fact that large duct type ductal adenocarcinomas are almost by definition well-differentiated tumors and would fall into Grade 1 category in the recently proposed grading scheme ${ }^{22}$ and others. ${ }^{23,24}$ These differences were not statistically significant; however, the analysis may be somewhat underpowered due to the low number of large duct type ductal adenocarcinoma cases. Large duct type ductal adenocarcinoma also has a predilection for tail localization when compared with ductal adenocarcinoma, which is also higher compared with the published data. ${ }^{5}$ In our study, the ratio of females versus males was higher in large duct type ductal adenocarcinoma when compared with ductal adenocarcinoma. This may be important in terms of the differential diagnosis between large duct type ductal adenocarcinomas and mucinous-cystic neoplasia, which are seen in females and involve the tail of the pancreas. As opposed to mucinous-cystic neoplasia, large duct type ductal adenocarcinomas are seen in an older population. Furthermore, females with large duct type ductal adenocarcinoma were even older (mean, 70). This may be helpful in distinguishing large duct type ductal adenocarcinomas 
from mucinous-cystic neoplasia (mean age, 48). As discussed before; however, although select sections of large duct type ductal adenocarcinoma at microscopic level may mimic; if the gross findings and the entire case is evaluated in the right context, this distinction should not be difficult.

In their study of pancreatic ductal adenocarcinomas with cystic features, which included examples of large duct type ductal adenocarcinoma in addition to cases with massive cystic degeneration of ordinary ductal adenocarcinoma, Kosmahl et $a l^{5}$ demonstrated that these tumors stain for CEA (83\%), MUC1 (71\%), MUC5AC (74\%), and MUC6 $(52 \%)$, and failed to stain for MUC2, supporting the neoplastic nature of these large ducts. We have shown in our study that there are also no significant differences between large duct type ductal adenocarcinoma and ductal adenocarcinoma in the immunohistochemical expression of p53, her2-neu, and mutational analysis of codon 12 of KRAS. These findings are consistent with the fact that large duct type ductal adenocarcinomas belong to ductal adenocarcinomas, and therefore have a common biology with similar molecular alterations to ordinary ductal adenocarcinoma.

In conclusion, the importance of large duct type ductal adenocarcinoma resides in its capacity to mimic, microscopically, especially in small tissue samples, non-invasive cystic and papillary lesions of the pancreas. As we are more and more often required to make a diagnosis of carcinoma on small biopsies, an awareness of this entity will result in an increased diagnostic accuracy.

\section{Acknowledgement}

This study is supported in part by the Georgia Cancer Coalition Distinguished Cancer Clinicians and Scientists Program, GA, USA.

\section{Disclosure/conflict of interest}

The authors declare no conflict of interest.

\section{References}

1 Chen J, Baithun SI. Morphological study of 391 cases of exocrine pancreatic tumours with special reference to the classification of exocrine pancreatic carcinoma. J Pathol 1985;146:17-29.

2 Hruban RH, Boffetta P, Hiraoka N, et al. Ductal adenocarcinoma of the pancreas In: Bosman FT, Carneiro F, Hruban RH, Theise ND (eds). World Health Organization Classification of Tumors of the Digestive System 4 edn, Vol., IARC Press: Lyon, 2010, pp 279-334.

3 Hruban RH, Pitman MB, Klimstra DS. Ductal adenocarcinoma In: Silverberg SG (ed). AFIP Atlas of Tumor
Pathology Tumors of the Pancreas 4 edn, Vol., ARP Press: Washington, DC, 2007, pp 111-164.

4 Adsay NV, Klimstra DS. Cystic forms of typically solid pancreatic tumors. Semin Diagn Pathol 2000;17:66-81.

5 Kosmahl M, Pauser U, Anlauf M, et al. Pancreatic ductal adenocarcinomas with cystic features: neither rare nor uniform. Mod Pathol 2005;18: 1157-1164.

6 Adsay NV. Cystic lesions of the pancreas. Mod Pathol 2007;20:71-93.

7 Adsay NV, Klimstra DS. Pancreatic tumors and pancreatic resection. Pathology and classification of pancreatic and ampullary tumors In: Blumgart LH (ed). Surgery of the Liver, Biliary Tract and Pancreas 4 edn, Vol., Elsevier: Philadelphia, 2007, pp 829-849.

8 Adsay NV, Klimstra DS. Tumors of the Pancreas and Ampulla of Vater In: Odze RD, Goldblum JR (eds). Surgical Pathology of the GI Tract, Liver. Biliary Tract, and Pancreas 2 edn, Vol., Elsevier: Philadelphia, 2009, pp 909-960.

9 Basturk O, Coban I, Adsay NV. Pancreatic cysts: pathologic classification, differential diagnosis, and clinical implications. Arch Pathol Lab Med 2009;133: 423-438.

10 Kloppel G. Clinicopathologic view of intraductal papillary-mucinous tumor of the pancreas. Hepatogastroenterology 1998;45:1981-1985.

11 Sessa F, Solcia E, Capella C, et al. Intraductal papillary-mucinous tumours represent a distinct group of pancreatic neoplasms: an investigation of tumour cell differentiation and K-ras, p53 and c-erbB-2 abnormalities in 26 patients. Virchows Arch 1994; 425:357-367.

12 Compagno J, Oertel JE. Mucinous cystic neoplasms of the pancreas with overt and latent malignancy (cystadenocarcinoma and cystadenoma). A clinicopathologic study of 41 cases. Am J Clin Pathol 1978;69: 573-580.

13 Thompson LDR, Becker RC, Pryzgodski RM, et al. Mucinous cystic neoplasm (mucinous cystadenocarcinoma of low malignant potential) of the pancreas: A clinicopathologic study of 130 cases. Am J Surg Pathol 1999;23:1-16.

14 Wilentz RE, Albores-Saavedra J, Hruban RH. Mucinous cystic neoplasms of the pancreas. Semin Diagn Pathol 2000;17:31-43.

15 Zamboni G, Scarpa A, Bogina G, et al. Mucinous cystic tumors of the pancreas: clinicopathological features, prognosis, and relationship to other mucinous cystic tumors. Am J Surg Pathol 1999;23:410-422.

16 Andea A, Sarkar F, Adsay NV. Clinicopathological correlates of pancreatic intraepithelial neoplasia: a comparative analysis of 82 cases with and 152 cases without pancreatic ductal adenocarcinoma. Mod Pathol 2003;16:996-1006.

17 Hruban RH, Adsay NV, Albores-Saavedra J, et al. Pancreatic intraepithelial neoplasia: a new nomenclature and classification system for pancreatic duct lesions. Am J Surg Pathol 2001;25:579-586.

18 Edge SB, Byrd DR, Compton CC, et al. Exocrine and Endocrine Pancreas AJCC Cancer Staging Manual, 7th edn, Vol Springer, 2010, pp 285-296.

19 Sarkar FH, Valdivieso M, Borders J, et al. A universal method for the mutational analysis of K-ras and p53 gene in non-small-cell lung cancer using formalinfixed paraffin-embedded tissue. Diag Mol Pathol 1995;4:266-273. 
20 Azar C, Van de Stadt J, Rickaert F, et al. Intraductal papillary mucinous tumours of the pancreas. Clinical and therapeutic issues in 32 patients. Gut 1996; 39:457-464.

21 Evans JD, Morton DG, Neoptolemos JP. Chronic pancreatitis and pancreatic carcinoma. Postgrad Med J 1997;73:543-548.

22 Adsay NV, Basturk O, Bonnett M, et al. A proposal for a new grading scheme for pancreatic ductal adenocarcinoma (DA): application of a practical and prognostically relevant system similar to Gleason scoring. Am J Surg Pathol 2005;29:724-733.
23 Klöppel G, Lingenthal G, von Bülow $\mathrm{M}$, et al. Histological and fine structural features of pancreatic ductal adenocarcinomas in relation to growth and prognosis: studies in xenografted tumours and clinicohistopathological correlation in a series of 75 cases. Histopathology 1985;9:841-856.

24 Lüttges J, Schemm S, Vogel I, et al. The grade of pancreatic ductal carcinoma is an independent prognostic factor and is superior to the immunohistochemical assessment of proliferation. J Pathology 2000;191: 154-161. 